\title{
On the curvature of free boundaries with a Bernoulli-type condition
}

\author{
Simone Cecchini*, Rolando Magnanini \\ Università degli Studi di Firenze, Dipartimento di Matematica “Ulisse Dini”, viale Morgagni 67, I-50134, Firenze, Italy
}

Received 4 April 2006; accepted 28 November 2006

\begin{abstract}
In this paper we study the classical external Bernoulli problem set in an annular domain $\Omega$ of the plane.

We focus on the curvature of the free boundary $\Gamma$ (outer component of the boundary of our domain) and establish a one-to-one correspondence between positive/negative curvature arcs of $\Gamma$ and of the curve $\gamma$ representing the data, extending a method put forward by A. Acker. Moreover we show that the positive curvature arcs on the free boundary bend less than the corresponding arcs on the inner curve, i.e. the maximum attained by the curvature is greater on $\gamma$ than on $\Gamma$. Thus we can draw the following conclusions: the geometry of $\Gamma$ is simpler than that of $\gamma$ (an already known result); the shape of $\Gamma$ is alleviated with respect to that of $\gamma$.
\end{abstract}

(c) 2006 Elsevier Ltd. All rights reserved.

MSC: 35R35; 35B99; 76D27

Keywords: Bernoulli's problem; Free boundaries; Qualitative properties

\section{Introduction}

The Bernoulli free boundary problem has applications to fluid dynamics, optimal insulation for heat or electric drilling and galvanization [1]. In a typical situation, one wants to design an annular condenser in which one of the plates is prescribed while the other has to be determined in such a way that the electrostatic field intensity remains constant on it. Depending on whether we prescribe the inner or outer plate we have an exterior or interior Bernoulli problem.

Besides Beurling's seminal paper [2], in which he pointed out the substantial difference between interior and exterior problem and the distinction between elliptic and hyperbolic solutions, we refer the reader to Acker's works [3,4], Flucher and Rumpf's [1] and the references therein. In particular, the latter contains a more rigorous definition of elliptic and hyperbolic solutions and gives a good presentation of old and new results concerning existence, uniqueness, regularity and qualitative properties of the free boundary, together with effective numerical algorithms.

In this paper, we shall consider the exterior Bernoulli problem in the plane, that can be stated as follows. Given a simple closed curve $\gamma$, find an external curve $\Gamma$ such that $\Gamma$ and $\gamma$ bound an annular domain $\Omega$, whose capacity

\footnotetext{
* Corresponding author. Tel.: +39 055 4237486; fax: +39 0554222695.

E-mail addresses: simone.cecchini@math.unifi.it (S. Cecchini), rolando.magnanini@math.unifi.it (R. Magnanini).
} 
potential $u$, determined by the conditions

$$
\begin{aligned}
& \Delta u=0 \quad \text { in } \Omega, \\
& u=1 \quad \text { on } \gamma, \\
& u=0 \quad \text { on } \Gamma,
\end{aligned}
$$

also satisfies

$$
|\nabla u|=1 \text { on } \Gamma,
$$

where $\nabla u$ denotes the gradient of $u$.

Proof of the existence of such a $\Gamma$ can be found in the already cited Beurling paper [2], in which the author proves that an annular domain satisfying our problem can always be determined. Thus we may assume that $\Gamma$ is a connected, simple curve. Moreover, Lewy [5] proved that $\Gamma$ is analytic (see also [6]).

Our aim is to describe the free boundary $\Gamma$ in terms of its curvature; namely, we shall show how the curvature of $\gamma$ determines that of $\Gamma$. To carry out our analysis, we will adapt the methods exposed in Acker's papers $[3,4]$ to the modified curvature functions

$$
h^{*}=\frac{h}{|\nabla u|} \quad \text { and } \quad k^{*}=\frac{k}{|\nabla u|}
$$

(where $h$ and $k$ are the curvatures of the level curves and of the curves of steepest descent of $u$ respectively) which happen to be harmonic conjugate in $\Omega$, as observed by Talenti [7]. We will show in Section 2 that $h^{*}$ and $k^{*}$ are, respectively, the curvatures of the level curves and of the curves of steepest descent of $u$ in the conformal metric

$$
(\mathrm{d} l)^{2}=|\nabla u(x, y)|^{2}\left[(\mathrm{~d} x)^{2}+(\mathrm{d} y)^{2}\right] .
$$

Moreover, in such a metric, level curves and curves of steepest descent are geodesic.

In Theorems 1-3 we assume that an elliptic solution $\Gamma$ of problems (1)-(4) is at hand. As already mentioned, $\Gamma$ is a connected simple analytic curve.

Theorem 1. Let $\left\{z_{1}, z_{2}, \ldots, z_{n}\right\}$ be an enumeration of distinct points of $\Gamma$, whose subscripts are given according to their order of occurrence on $\Gamma$, counterclockwise.

Suppose that each $z_{i}$ satisfies one of the following properties:

(i) the value of $h^{*}$ at $z_{i}$ is a local minimum on $\Gamma$;

(ii) the value of $h^{*}$ at $z_{i}$ is a local maximum on $\Gamma$;

(iii) on $\Gamma$, the sign of $h^{*}$ changes at $z_{i}$, turning from positive to negative;

(iv) on $\Gamma$, the sign of $h^{*}$ changes at $z_{i}$, turning from negative to positive.

Then there exists a collection $\left\{\zeta_{1}, \zeta_{2}, \ldots, \zeta_{n}\right\}$ of sub-arcs of $\gamma$, possibly degenerating to points, such that $h^{*}$ is constant on $\zeta_{i}$ and satisfies (i)-(iv) on $\gamma$, respectively, with $z_{i}$ replaced by $\zeta_{i}$.

Moreover, if $z_{i}$ satisfies (i) or (ii), then $h^{*}>h^{*}\left(z_{i}\right)$ or $h^{*}<h^{*}\left(z_{i}\right)$ on $\zeta_{i}$, respectively.

This result is not completely satisfactory. In fact, while the changes of sign of $h^{*}$ and $h$ coincide on both $\gamma$ and $\Gamma$, their extremal points are the same on $\Gamma$ but not on $\gamma$. Theorems 2 and 3 below takes this observation into account.

Given a curve $\beta$, we define a collection $\left\{\beta_{1}, \beta_{2}, \ldots, \beta_{m}\right\}$ of sub-arcs of $\beta$ as a partition when the $\beta_{i}$ have pairwise disjoint interiors and $\bigcup_{i=1}^{m} \beta_{i}=\beta$.

Theorem 2. Let $\left\{\Gamma_{1}, \Gamma_{2}, \ldots, \Gamma_{m}\right\}$ be a partition of $\Gamma$ made of arcs which are maximal with respect to the property that for each $i \in\{1,2, \ldots, m\}$, one and only one of the following holds:

(i) $h(z)>0$ for all $z \in \Gamma_{i}$;

(ii) $h(z)<0$ for all $z \in \Gamma_{i}$;

(iii) $h(z)=0$ for all $z \in \Gamma_{i}$;

Then for each $i \in\{1,2, \ldots, m\}$ we can determine a sub-arc $\gamma_{i} \subset \gamma$ which has the same property as $\Gamma_{i}$. Moreover the arcs $\gamma_{i}$ are mutually disjoint. 
As a by-product of Theorem 2, we can infer a classical result of Tepper [8]: if $\gamma$ is convex, then also $\Gamma$ is convex.

Theorem 3. Let $\left\{z_{1}, z_{2}, \ldots, z_{n}\right\}$ be given as in Theorem 1. Suppose that each $z_{i}, 1 \leq i \leq n$, satisfies one of the following properties:

(i) the value of $h$ at $z_{i}$ is a non-negative local maximum on $\Gamma$;

(ii) on $\Gamma$ the sign of $h$ changes at $z_{i}$, turning from positive to negative;

(iii) on $\Gamma$ the sign of $h$ changes at $z_{i}$, turning from negative to positive.

Then there exists a collection $\left\{\zeta_{1}, \zeta_{2}, \ldots, \zeta_{n}\right\}$ of sub-arcs of $\gamma$, possibly degenerating to points, such that each $\zeta_{i}$ satisfies:

(a) if (i) holds, then $h>h\left(z_{i}\right) \cdot \mathrm{e}^{h\left(z_{i}\right)}$ on $\zeta_{i}$; moreover $z_{i}$ is joined to $\zeta_{i}$ by a level curve $\{k=0\}$;

(b) if (ii) or (iii) holds, then (ii) or (iii) holds for $h$ on $\gamma$, with $z_{i}$ replaced by $\zeta_{i}$.

By studying the argument of $\nabla u$ Acker proves that to each point of $\Gamma$ at which $h$ is strictly positive, negative or changes sign there corresponds a point of $\gamma$ at which $h$ is positive, negative or changes sign with the same transition, respectively [4, Theorem 4].

By studying $h^{*}$, we improve Acker's results to the extent that to each sub-arc $\Gamma_{i}$ of $\Gamma$ which is maximal with respect to the property that $h$ does not change sign on it, there corresponds a sub-arc $\gamma_{i}$ of $\gamma$ that is maximal with respect to the same property. Moreover, by Theorem 3 we can conclude that the positive maximum of $h$ on $\gamma_{i}$ is greater than the maximum of $h$ on $\Gamma_{i}$, i.e. $\Gamma_{i}$ bends less then $\gamma_{i}$. Thus we can deduce not only that the geometry of $\Gamma$ is simpler than that of $\gamma$ (as we can do from Acker's papers), but also that the protuberances of $\gamma$ directed away from the inside are alleviated in passing from $\gamma$ to $\Gamma$.

We begin with some preparatory results in Section 2. The proofs of Theorems 1-3 will be presented in Section 3. Two appendices collect the proofs of two technical lemmas (Lemmas 12 and 17).

\section{Curvature functions}

Let $\Omega$ be an open subset of $\mathbb{R}^{2}$ and suppose that $u \in C^{2}(\Omega)$. If $\nabla u(z) \neq 0$ for $z \in \Omega$, we can define $h(z)$ and $k(z)$, respectively, as the signed curvatures of the (unique) level curve and of the (unique) curve of steepest descent of $u$ passing through $z$. We choose the sign of $h(z)$ and $k(z)$ so that $h(z) \cdot \nabla u(z)$ and $k(z) \cdot \nabla u(z)$ are always directed towards the respective centers of curvature.

We recall the following result due to Talenti [7] (as usual we define Wirtinger's operators as $\partial u=(1 / 2)\left(u_{x}-\right.$ $\left.\left.\mathrm{i} u_{y}\right), \bar{\partial} u=(1 / 2)\left(u_{x}+\mathrm{i} u_{y}\right)\right)$.

Theorem A. Let $u$ be a real-valued function of class $C^{2}(\Omega)$. If $\nabla u \neq 0$ in $\Omega$, then

(i) $h=-\operatorname{div} \frac{\nabla u}{|\nabla u|}, k=-\operatorname{div}\left(\begin{array}{cc}0 & 1 \\ -1 & 0\end{array}\right) \frac{\nabla u}{|\nabla u|}$;

(ii) if $u$ is harmonic, $\frac{h+\mathrm{i} k}{|\nabla u|}$ is a holomorphic function.

Extensions of such results to the case in which $u$ has a finite number of critical points can be found in Alessandrini and Magnanini [9].

Now we investigate a metric feature of the curvature functions $h^{*}$ and $k^{*}$. Let us consider on $\Omega$ the Riemannian metric given by (6). By direct inspection of (6) we conclude that $\mathrm{d} l^{2}$ is conformal (see [10]); therefore measures of angles with respect to it are equal to Euclidean ones. We denote by $h^{\#}$ the curvature of level lines with respect to $\mathrm{d} l^{2}$, determined as follows: for $z \in \Omega$ we consider any parametrization $t \mapsto z^{\#}(t) \in \mathbb{C}$ of the level curve of $u$ through $z$ which is natural with respect to $\mathrm{d} l^{2}$. Let $\phi^{\#}(t)$ be a locally continuous determination of the argument of the tangent unit vector as determined in $\mathrm{d} l^{2}$. By the previous remark, $\phi^{\#} \equiv \phi$ (where $\phi$ is a local determination of the argument of the tangent with respect to the Euclidean metric); then we define

$$
h^{\#}(z)=\frac{\mathrm{d} \phi^{\#}}{\mathrm{~d} t}=\frac{\mathrm{d} \phi}{\mathrm{d} t} .
$$

Analogous meaning is to be given to $k^{\#}$. We state the following: 
Proposition 4. Let $u \in C^{2}(\Omega)$. For all $z \in \Omega$, we have

$$
h^{\#}(z)=h^{*}(z), \quad k^{\#}(z)=k^{*}(z) .
$$

Proof. We limit ourselves to considering $h^{\#}$, the case of $k^{\#}$ being analogous. Let $z \in \bar{\Omega}$ and $z^{\#}(t), z(s)$ be parametrizations by arc length of the level curve through $z$ with respect to $\mathrm{d} l^{2}$ and the Euclidean metric respectively. Then we have

$$
h^{\#}(z)=\frac{\mathrm{d} \phi^{\#}}{\mathrm{~d} t}(z)=\frac{\mathrm{d} \phi}{\mathrm{d} s}(z) \cdot \frac{\mathrm{d} s}{\mathrm{~d} t}=h(z) \cdot \frac{\mathrm{d} s}{\mathrm{~d} t} .
$$

From (6) we infer that $\frac{\mathrm{d} t}{\mathrm{~d} s}=|\nabla u|$.

Now, let $\Omega$ be an annular domain bounded by regular, simple, closed curves $\Gamma$ and $\gamma$, and $u$ : $\Omega \rightarrow R$ be a solution of (1)-(4). Then we can regard $\gamma$ as a given curve and $\Gamma$ as a free boundary. From now on, unless otherwise specified, these are to be considered as the underlying assumptions.

On account of the topology of the set $\Omega$, we have to remove a curve of steepest descent $\beta$ from it, in order to obtain a single-valued function $v: \Omega \backslash \beta \rightarrow \mathbb{R}$, harmonic conjugate to $u$.

From the boundary conditions it follows [9] that $|\nabla u| \neq 0$ in $\bar{\Omega}$; therefore we can define a conformal map of the $z$-plane onto the $w$-plane, $\mathcal{G}: \Omega \backslash \gamma \rightarrow(0, C) \times(0,1)$ by $w=\mathcal{G}(z)=\mathrm{i} u(z)-v(z)$ (where $C$ is the length of $\Gamma$ ). By functional inversion and analytic extension, we come to a $C$-periodic, holomorphic function $\mathcal{F}$ of the strip $\mathrm{S}=\mathbb{R} \times(0,1)$ onto $\Omega$, which can be extended continuously to $\mathbb{R} \times[0,1]$ (actually analytically to $\mathbb{R} \times[0,1)$ ). Whenever it makes sense, $\mathcal{F}=\mathcal{G}^{-1}$ (thus $\left|\mathcal{F}^{\prime}\right| \neq 0$ in $\mathbb{R} \times[0,1)$ ). Moreover $\mathcal{F}$ maps $L=\mathbb{R} \times\{0\}$ and $l=\mathbb{R} \times\{1\}$ counterclockwise onto $\Gamma$ and $\gamma$ respectively.

Along with $\mathcal{F}$ we consider the harmonic functions $\rho, \theta: \mathrm{S} \rightarrow \mathbb{R}$ defined so as to satisfy $\rho+\mathrm{i} \theta=\log \mathcal{F}^{\prime}$.

By the boundary conditions on $u$ we easily find:

Proposition 5. $\rho \equiv 0$ and $\frac{\partial \theta}{\partial u} \equiv 0$ on $L$.

Let $h(z), k(z)$ be the curvatures of the level line and of the curve of steepest descent of $u$ through $z \in \Omega$ respectively, as defined above.

We set some notation. For all $\lambda \in[0,1]$ and $\alpha \in \mathbb{R}$, let $\Gamma^{\lambda}=\{z \in \bar{\Omega}: u(z)=\lambda\} ; z_{\lambda}: \mathcal{I}_{\lambda} \rightarrow \Gamma^{\lambda}$ be a parametrization by arc length of $\Gamma^{\lambda} ; \xi_{\alpha}(w)=w+\mathrm{i} \alpha$. Then, for any pair of level lines $\Gamma^{\lambda}$, $\Gamma^{\mu}$ we obtain a parametrization $P_{\lambda, \mu}: \mathcal{I}_{\lambda} \rightarrow \Gamma^{\mu}$ of $\Gamma^{\mu}$ by

$$
P_{\lambda, \mu}(t)=\mathcal{F} \circ \xi_{\mu-\lambda} \circ \mathcal{G} \circ z_{\lambda}(t) .
$$

This allows us to parametrize $\Gamma^{\mu}$ by the arc length parameter of $\Gamma^{\lambda}$. Taking $\lambda=0$ in the above formula, we find a way of interpreting $h^{*}$ geometrically. We express this in the following proposition.

Proposition 6. For any $z \in \Omega$, set $\mu=u(z)$. Given a local determination $\phi_{\mu}$ of the argument of $P_{0, \mu}^{\prime}$, it holds that

$$
h^{*}(z)=\frac{\mathrm{d} \phi_{\mu}}{\mathrm{d} t}(z)
$$

i.e. we determine $h^{*}$ at points of $\Gamma^{\mu}$ by differentiating $\phi_{\mu}$ with respect to the arc length of the free boundary $\Gamma$.

We are giving no proof here, Proposition 6 being a straightforward consequence of Proposition 9 below.

Observe that in general the curves of steepest descent of any $C^{2}(\Omega)$ function are geodesics in the metric $\mathrm{d} l^{2}$ defined by (6). Since $\mathrm{d} l^{2}$ can be regarded as the pull-back by $\mathcal{G}$ of the Euclidean metric (so that, e.g., to determine the length of a curve $\beta$, we have to evaluate the Euclidean length of its image $\mathcal{G} \circ \beta$ ), we can draw at once the following conclusions.

\section{Proposition 7. If $u$ is harmonic then}

(i) its level curves are geodesics with respect to $\mathrm{d} l^{2}$;

(ii) a parametrization by arc length of a level curve (a curve of steepest descent) of $u$ with respect to $\mathrm{d} l^{2}$ can be obtained as

$$
z_{\mu}^{\#}(t)=\mathcal{F}(t+\mathrm{i} \mu) \quad\left(z_{v}^{\#}(t)=\mathcal{F}(v+\mathrm{i}(1-t))\right) .
$$


Remark 8. Since $\mathcal{F}$ is conformal, $h^{*} \circ \mathcal{F}$ and $k^{*} \circ \mathcal{F}$ are harmonic functions in $\overline{\mathrm{S}}$. They will still be denoted by $h^{*}$ and $k^{*}$, the distinction of cases relying upon the specification of variables (e.g. $h^{*}(z)$ or $\left.h^{*}(w)\right)$.

We end this section with two other formulas for $h^{*}$ and $k^{*}$. These will be repeatedly used in the sequel.

Proposition 9. For all $w \in \overline{\mathrm{S}}$, we have that

$$
h^{*}(w)=\frac{\partial \theta}{\partial v}(w), \quad k^{*}(w)=-\frac{\partial \theta}{\partial u}(w) .
$$

Proof. Let $z \in \bar{\Omega}$ be such that $z \in \Gamma^{\mu}$ and $z_{\mu}^{\#}(t)=\mathcal{F}(t+\mathrm{i} \mu)$ be an arc length parametrization with respect to $\mathrm{d} l^{2}$. As $\theta=\arg \left(\mathcal{F}^{\prime}\right)=-\arg \left(\mathcal{G}^{\prime}\right)=-\arg (2 \mathrm{i} \partial u)=\arg (\nabla u)-(\pi / 2)=\arg \left(\left(z_{\mu}^{\#}\right)^{\prime}\right)=\phi_{\mu}$ we conclude that

$$
h^{*}=\frac{\mathrm{d} \phi_{\mu}}{\mathrm{d} t}(\mathcal{F}(w))=\frac{\mathrm{d} \theta}{\mathrm{d} t}(w)=\frac{\partial \theta}{\partial v}(w) .
$$

Corollary 10. Let $v$ be the external unit normal vector to $\Gamma$. Then, on $\Gamma$

(i) $k^{*} \equiv 0$,

(ii) $\frac{\partial h^{*}}{\partial v} \equiv 0$.

Proof. By Proposition 5 we get (i). By (i) and the Cauchy-Riemann equations we get (ii).

\section{Curvature of free boundaries}

In order to prove Theorems 1-3 we need some lemmas. These are mostly of a topological flavour and fulfill the same need as [3, Lemma 2]; actually they are nothing more than a rephrasing of that, but owing to the fact that we carry out our analysis in $\Omega$ (instead of the strip S), we have to expand a little more on this topic.

To begin with, we give the following definition.

Definition 11. Let $a \in \mathbb{R}$ and $z_{0} \in \bar{\Omega}$ be such that $h^{*}\left(z_{0}\right)<a\left(h^{*}\left(z_{0}\right)>a\right)$. By $\omega^{-}\left(z_{0}, a\right)\left(\omega^{+}\left(z_{0}, a\right)\right)$ we mean the component of the set

$$
\left\{z \in \bar{\Omega}: h^{*}(z)<a\right\} \quad\left(\left\{z \in \bar{\Omega}: h^{*}(z)>a\right\}\right)
$$

to which $z_{0}$ belongs.

We also set $B\left(z_{0}, r\right)=\left\{z \in \mathbb{C}:\left|z-z_{0}\right|<r\right\}$.

Given any Jordan curve $\beta \subset \mathbb{C}, D_{b}(\beta), D_{u}(\beta)$ indicate respectively the bounded (inner) and unbounded (outer) component of the complement of $\beta$.

The proof of the next lemma may be found in Appendix A.

Lemma 12. Let $c \subset \bar{\Omega}$ be a simple curve with endpoints $z_{1}, z_{2}, z_{1} \neq z_{2}, z_{1}, z_{2} \in \Gamma$. Then $z_{1}, z_{2}$ split $\Gamma$ into two arcs $\Gamma^{\prime}$ and $\Gamma^{\prime \prime}$ such that $\Gamma^{\prime} \cup \Gamma^{\prime \prime}=\Gamma, \Gamma^{\prime} \cap \Gamma^{\prime \prime}=\left\{z_{1}, z_{2}\right\}$ and $D_{b}(\gamma)$ is contained either in $\Omega^{\prime}=D_{b}\left(c \cup \Gamma^{\prime}\right)$ or in $\Omega^{\prime \prime}=D_{b}\left(c \cup \Gamma^{\prime \prime}\right)$.

We are now ready to establish the relevant topological properties of components $\omega^{-}\left(z_{0}, a\right)$. We set apart the most delicate ones to prove in the following lemma.

Lemma 13. If $a$ is a value of $h^{*}$ in $\Gamma$ and $z_{0} \in \Gamma$ is chosen such that $h^{*}\left(z_{0}\right)<a$ then

(i) $\omega^{-}\left(z_{0}, a\right)$ is simply connected;

(ii) let $c \subset \omega^{-}\left(z_{0}, a\right)$ be a simple curve with endpoints $z_{1}, z_{2} \in \Gamma, z_{1} \neq z_{2}$ :

if $D_{b}(\gamma) \subseteq D_{b}\left(c \cup \Gamma^{\prime}\right)$ then $\Gamma^{\prime \prime} \subset \omega^{-}\left(z_{0}, a\right)$;

if $D_{b}(\gamma) \subseteq D_{b}\left(c \cup \Gamma^{\prime \prime}\right)$ then $\Gamma^{\prime} \subset \omega^{-}\left(z_{0}, a\right)$. 
Proof. (i) Let $\beta$ be a simple, closed curve in $\omega^{-}\left(z_{0}, a\right)$. Then $h^{*}<a$ on $\beta$. There are the alternatives: either $D_{b}(\beta) \subset \bar{\Omega}$ or $D_{b}(\beta) \nsubseteq \bar{\Omega}$. In the former case, $h^{*}$ being harmonic in the interior of $D_{b}(\beta)$, we conclude by the maximum principle that for all $z \in D_{b}(\beta), h^{*}(z)<a$. Therefore $D_{b}(\beta) \subset \omega^{-}\left(z_{0}, a\right)$. In the latter case $\beta$ encircles $D_{b}(\gamma)$ so $D_{b}(\gamma) \subset D_{b}(\beta)$ and $D_{u}(\beta) \subset D_{u}(\gamma)$. It follows that $\Omega^{\prime}=D_{b}(\Gamma) \cap D_{u}(\beta) \subset \Omega$ and $\partial \Omega^{\prime}=\Gamma \cup \beta$. By Hopf's lemma, the harmonic function $h^{*}$ cannot attain its maximum on $\Omega^{\prime}$ at points of $\Gamma$, for there $\left(\partial h^{*} / \partial v\right) \equiv 0$ (see Corollary 10). It follows that the maximum of $h^{*}$ has to be found in $\beta$. As $\beta \subset \omega^{-}\left(z_{0}, a\right)$, it holds that $h^{*}<a$ in $\Omega^{\prime}$. But then we reach a contradiction: from our hypothesis we know that there exists $z^{*} \in \Gamma$ such that $h^{*}\left(z^{*}\right)=a$, but then $z^{*}$ would maximize $h^{*}$ on $\Gamma$.

(ii) We suppose that $D_{b}(\gamma) \subset D_{b}\left(c \cup \Gamma^{\prime \prime}\right)$.

Then $\partial D_{b}\left(c \cup \Gamma^{\prime}\right)=c \cup \Gamma^{\prime}$. Since $\left(\partial h^{*} / \partial v\right) \equiv 0$ on $\Gamma$, the maximum of $h^{*}$ on $D_{b}\left(c \cup \Gamma^{\prime}\right)$ is attained at some inner point of $c$. It follows that $\max \left\{h^{*}(z): z \in \omega^{-}\left(z_{0}, a\right)\right\}<a$ and therefore $h^{*}<a$ on $\Gamma^{\prime}$, i.e. $\Gamma^{\prime} \subset \omega^{-}\left(z_{0}, a\right)$.

Analogous statements hold for components $\omega^{+}\left(z_{0}, a\right)$.

We need some more notation. Let $z_{1}, z_{2} \in \Gamma^{\mu}$. We write $z_{1} \prec z_{2}\left(z_{1} \preceq z_{2}\right)$ if for every orientation preserving local parametrization $p_{\mu}:(0,1) \rightarrow \Gamma^{\mu}$ such that $z_{1}, z_{2} \in p((0,1)), p\left(t_{1}\right)=z_{1}, p\left(t_{2}\right)=z_{2}$ we have $t_{1}<t_{2}\left(t_{1} \leq t_{2}\right)$. If $z_{1} \prec z_{2}$ we denote by $\left[z_{1}, z_{2}\right]$ the $\operatorname{arc}\left\{z \in \Gamma^{\mu}: z_{1} \preceq z \preceq z_{2}\right\}$. If $A_{1}, A_{2} \subset \Gamma^{\mu}$ are two arcs, we write $A_{1} \prec A_{2}$ $\left(A_{1} \preceq A_{2}\right)$ if for every pair $z_{1} \in A_{1}, z_{2} \in A_{2}$ it holds that $z_{1} \prec z_{2}\left(z_{1} \preceq z_{2}\right)$.

In Lemma 14 we are setting $\omega=\omega^{-}\left(z_{0}, a\right)$ and we denote by $b(\omega)$ the boundary of $\omega$ relative to $\bar{\Omega}$. Notice that due to the fact that $\Gamma$ is an analytic curve - (iii) entails that either $b(\omega) \cap \Gamma$ is a discrete set, or $h^{*}$ is constant on $\Gamma$ and thus in $\Omega$; observe also that some parts of $b(\omega)$ may lie on $\gamma$.

Lemma 14. Let a be a value of $h^{*}$ on $\Gamma$ and suppose that $z_{0} \in \Gamma$ is such that $h^{*}\left(z_{0}\right)<a$. Then

(i) $\omega$ is relatively open in $\bar{\Omega}$ (and hence $\omega \cap \gamma$ is open as a subset of $\gamma$ );

(ii) $\omega$ is simply connected and $\omega \cap \Gamma$ is connected;

(iii) $h^{*} \equiv$ a on $b(\omega)$;

(iv) $\omega \cap \gamma \neq \varnothing$;

(v) $b(\omega)$ is locally an analytic simple arc, except at points where $\nabla h^{*}=0$;

(vi) if $b(\omega)$ is given an orientation such that $h^{*}<a\left(h^{*}>a\right)$ to the right, then $k^{*}$ is strictly decreasing (increasing) on $b(\omega)$.

Proof. (i), (iii) $h^{*}$ is indeed continuous in $\bar{\Omega}$.

(ii) We have already proved (see Lemma 13) that $\omega$ is simply connected. Now, if $z_{1}, z_{2} \in \omega \cap \Gamma$ are such that $z_{1} \neq z_{2}$, then we can find an arc $\beta \subset \omega$ connecting $z_{1}$ to $z_{2}$ (we recall that $\omega$ is arcwise connected). By an application of claim (ii) of Lemma 13 we conclude that one of the arcs $\Gamma^{\prime}$ and $\Gamma^{\prime \prime}$ is contained in $\omega$.

(iv) Let us suppose that $\omega \cap \gamma=\varnothing$ and try to reach a contradiction. We have $\partial \omega=(\Gamma \cap \omega) \cup b(\omega)$. From claim (iii) we know that $h^{*} \equiv a$ on $b(\omega)$. Since $\left(\partial h^{*} / \partial \nu\right) \equiv 0$ on $\Gamma$, neither the maximum nor the minimum of $h^{*}$ can be attained at points of $\Gamma$. Then $h^{*} \equiv a$ on $\bar{\omega}$, contrary to the definition of $\omega$.

(v) From claim (iii), $b(\omega)$ is a level curve of the analytic function $h^{*}$.

(vi) It is a straightforward consequence of the Cauchy-Riemann equations relating $h^{*}$ and $k^{*}$.

Corollary 15. Let $z_{1}, z_{2} \in \Gamma$. If $\left(\omega^{-}\left(z_{1}, a\right) \cup \omega^{-}\left(z_{2}, a\right)\right) \cap \Gamma$ is not connected, then $\omega^{-}\left(z_{1}, a\right) \cap \omega^{-}\left(z_{2}, a\right)=\varnothing$.

Proof. We have to show that $\omega^{-}\left(z_{1}, a\right)$ and $\omega^{-}\left(z_{2}, a\right)$ are distinct. By claim (ii) of Lemma 14, if $\omega^{-}\left(z_{1}, a\right)=$ $\omega^{-}\left(z_{2}, a\right)$ then $\left(\omega^{-}\left(z_{1}, a\right) \cup \omega^{-}\left(z_{2}, a\right)\right) \cap \Gamma=\omega^{-}\left(z_{1}, a\right) \cap \Gamma$ is connected.

We can exploit the information given by Lemma 14 in two ways. We begin by proving Theorem 2 . As $\operatorname{sgn}(h) \equiv$ $\operatorname{sgn}\left(h^{*}\right)$ this can be phrased in terms of $h$ and proved by use of $h^{*}$.

Proof (Theorem 2). We consider the set partition of $\Gamma,\left\{\Gamma_{1}, \ldots, \Gamma_{n}\right\}$ given in the statement of the theorem at hand.

Since $\Gamma$ is analytic and compact, the set in which $h^{*}$ vanishes is finite. Hence, for each $i \in\{1, \ldots, n\}$, we have that $h^{*}>0$ on $\Gamma_{i}$ or $h^{*}<0$ on $\Gamma_{i}$ or $\Gamma_{i}$ reduces to a point.

For all $i$ we choose $z^{i} \in \Gamma_{i}$ and consider $\omega^{-}\left(z^{i}, 0\right)$ (or $\omega^{+}\left(z^{i}, 0\right)$ ). By Lemma 14, claim (iv), it holds that $\omega^{ \pm}\left(z^{i}, 0\right) \cap \gamma \neq \varnothing$. We denote by $\gamma_{i}$ one of the components of $\omega^{ \pm}\left(z^{i}, 0\right) \cap \gamma$. Since $i_{1} \neq i_{2}$ implies $\Gamma_{i_{1}} \cap \Gamma_{i_{2}}=\varnothing$, we have (see Corollary 15) $\omega^{ \pm}\left(z^{i_{1}}, 0\right) \cap \omega^{ \pm}\left(z^{i_{2}}, 0\right)$ and thus $\gamma_{i_{1}} \cap \gamma_{i_{2}}=\varnothing$. 


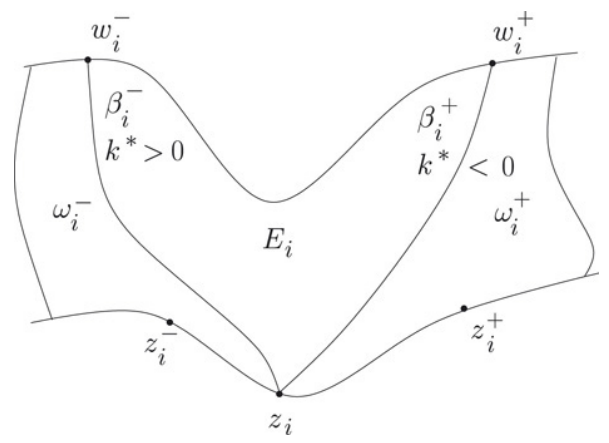

Fig. 1. The components $\omega_{i}^{-}$and $\omega_{i}^{+}$.

The proof of Theorem 1 is more delicate.

Proof (Theorem 1). This proof splits in two steps:

(a) for each $z_{i}$ there exists an arc $\zeta_{i}$ of $\gamma$, which shares the same property of $z_{i}$;

(b) if $z_{i_{1}} \neq z_{i_{2}}$ then $\zeta_{i_{1}} \cap \zeta_{i_{2}}=\varnothing$.

Were $h^{*} \equiv$ constant over $\bar{\Omega}$, the theorem would trivially hold, so we exclude this case in what follows. Consequently we can suppose that, if $h^{*}\left(z_{i}\right)=a$, there exists an $\operatorname{arc}\left[z_{i}^{-}, z_{i}^{+}\right] \subset \Gamma$ such that $z_{i}^{-}<z_{i}<z_{i}^{+}$and $h^{*}$ assumes the value $a$ on $\left[z_{i}^{-}, z_{i}^{+}\right]$only at $z_{i}$.

(a) First we consider the case of $a$ being a maximum for $h^{*}$. Since we can choose $z_{i}^{-}, z_{i}^{+} \in \Gamma$ as above, we have $h^{*}\left(z_{i}^{ \pm}\right)<a$. Put $\omega_{i}^{ \pm}=\omega^{-}\left(z_{i}^{ \pm}, a\right)$; then, in view of Lemma 14 we obtain the configuration sketched in Fig. 1, i.e. there exist two arcs $\beta_{i}^{-}, \beta_{i}^{+}$(these are indeed components of $b\left(\omega_{i}^{-}\right)$and $b\left(\omega_{i}^{+}\right)$), such that $\beta_{i}^{-} \cap \beta_{i}^{+}=\left\{z_{i}\right\}, h^{*} \equiv a$ on $\beta_{i}^{ \pm}$, $k^{*}\left(w_{i}^{-}\right)>0, k^{*}\left(w_{i}^{+}\right)<0$, where $w_{i}^{-}, w_{i}^{+}$are points at which $\gamma$ intersects $\beta_{i}^{-}$and $\beta_{i}^{+}$, respectively.

Let $E_{i}$ be the open set bounded by the $\operatorname{arcs} \beta_{i}^{-}, \beta_{i}^{+},\left[w_{i}^{-}, w_{i}^{+}\right]$. We cannot have $h^{*}(z) \leq a$ for all $z \in E_{i}$, for otherwise we could choose $w \in \beta_{i}^{-}$and a ball $B=B(w, \delta) \subset E_{i} \cup \omega_{i}^{-} \cup \beta_{i}^{-}$so that $h^{*}<a$ in $B \cap \omega_{i}^{-}, h^{*} \leq a$ on $B \cap\left(E_{i} \cup \beta_{i}^{-}\right)$. But then, by the mean value theorem for harmonic functions, we would get $h^{*}(w)<a$.

Let $D$ be a component of $\left\{z \in E_{i}: h^{*}(z)>a\right\}$. If $D \cap \gamma=\varnothing$, then $h^{*} \equiv a$ on $\partial D$ and so - by the maximum principle $-h^{*} \equiv a$ in $D$. But then $h^{*} \equiv a$ over the whole of $\bar{\Omega}$. So we can suppose that $D \cap \gamma \neq \varnothing$. Moreover (again by the maximum principle) there is at least one point $\zeta \in D \cap \gamma$ such that $h^{*}(\zeta)>a$. We set $\zeta_{i}$ to be the maximal arc containing $\zeta$ in which $h^{*} \equiv h^{*}(\zeta)$.

We stress that in this case $\zeta_{i} \subset\left[w_{i}^{-}, w_{i}^{+}\right]$since $h^{*}>a=h^{*}\left(z_{i}\right)$ while $h^{*}\left(w_{i}^{-}\right)=h^{*}\left(w_{i}^{+}\right)=a$ and $\zeta_{i} \subset E_{i} \cap \gamma$ since $h^{*} \equiv a$ on $\partial \omega_{i}^{ \pm}$.

If $a$ had been a minimum we could have dealt with it in an analogous fashion.

We conclude (a) by considering the case of $z_{i}$ being a sign transition. We choose again $z_{i}^{-}, z_{i}^{+} \in \Gamma$ such that $z_{i}^{-}<z_{i}<z_{i}^{+}, h^{*}$ assumes the value 0 on $\left[z_{i}^{-}, z_{i}^{+}\right]$only at $z_{i}$ and $h^{*}\left(z_{i}^{-}\right)<0, h^{*}\left(z_{i}^{+}\right)>0$ (or conversely). Then we consider the components $\omega_{i}^{-}=\omega^{-}\left(z_{i}^{-}, 0\right), \omega_{i}^{+}=\omega^{+}\left(z_{i}^{+}, 0\right)$. We know, by Lemma 14, claims (i) and (iv), that there exists $W_{i}^{ \pm} \in \omega_{i}^{ \pm} \cap \gamma$ such that $h^{*}\left(W_{i}^{-}\right)<0, h^{*}\left(W_{i}^{+}\right)>0$. By continuity, there exists a maximal arc $\zeta_{i}$ contained in the interior of $\left[W_{i}^{-}, W_{i}^{+}\right]$such that $h^{*} \equiv 0$ on $\zeta_{i}, h^{*}<0$ on a small arc immediately preceding $\zeta_{i}, h^{*}>0$ on a small arc immediately following $\zeta_{i}$. Actually many such sets may exist. In order to prove part (b) we need to make a suitable choice among them: we will explain our criterion later, in the proof of claim (b).

(b) Let us consider $z_{i-1}, z_{i} \in \Gamma$ both enjoying the property of being an extremum or a sign transition for $h^{*}$ on $\Gamma$. Whichever kind they are, we can exclude that $h^{*}\left(z_{i-1}\right)=h^{*}\left(z_{i}\right)$, for otherwise we might spot an extremum point $z^{*}$ such that $z_{i-1}<z^{*}<z_{i}$, and $h^{*}\left(z^{*}\right)>h^{*}\left(z_{i-1}\right)=h^{*}\left(z_{i}\right)$ or $h^{*}\left(z^{*}\right)<h^{*}\left(z_{i-1}\right)=h^{*}\left(z_{i}\right)$.

This remark allows us to limit our analysis to the two cases in which $z_{i-1}$ is an extremum and $z_{i}$ is either an extremum or a sign transition; both cases bearing the condition $h^{*}\left(z_{i-1}\right) \neq h^{*}\left(z_{i}\right)$. This last let us infer that $E_{i-1}$ and $E_{i}$ determined as in part (a) of the proof are disjoint. Indeed, should $E_{i-1} \cap E_{i}$ be non-empty, $\beta_{i-1}^{+}$and $\beta_{i}^{-}$would meet. (To have a proof of this assertion complying with the standards of rigor, consider the boundary relative to $\partial E_{i-1}$ of the set $E_{i} \cap \partial E_{i-1}$. This boundary is not empty - since $z_{i-1} \in \partial E_{i-1} \backslash E_{i}$, and thus $\partial E_{i-1} \cap E_{i} \neq \partial E_{i-1}-$ and is 


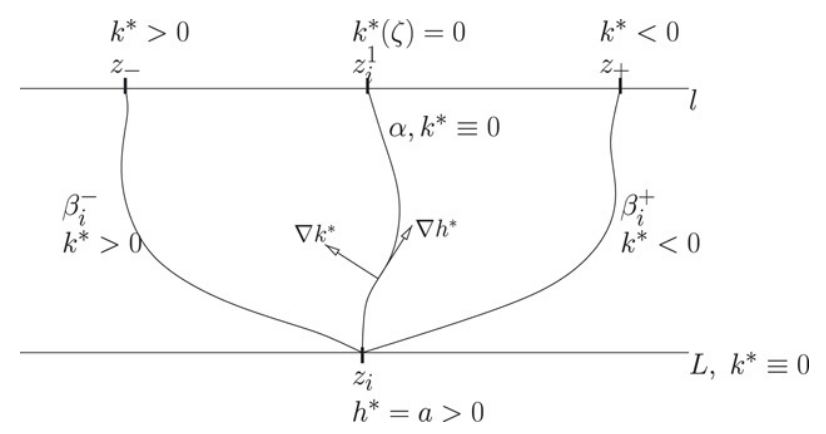

Fig. 2. How to determine $z_{i}^{1}$ for non-negative minimizers.

contained in $\partial E_{i-1} \cap \partial E_{i}$. Take into account that $\partial E_{i}$ is a simple analytic arc.) But $h^{*} \equiv h^{*}\left(z_{i-1}\right)$ on $\beta_{i-1}^{+}$, while $h^{*} \equiv h^{*}\left(z_{i}\right) \neq h^{*}\left(z_{i-1}\right)$ on $\beta_{i}^{-}$; thus we reach a contradiction.

Since in the case of both $z_{i-1}, z_{i}$ being extremum points, $\zeta_{i-1} \subset E_{i-1} \cap \gamma, \zeta_{i} \subset E_{i} \cap \gamma$, the remark above let us conclude.

The case of $z_{i-1}$ being an extremum, $z_{i}$ a sign transition (or the converse occurring) is the only relevant one left. Let us resume the construction of $\omega_{i}^{ \pm}$delineated in the first part of the proof. We are restraining our analysis to the case in which there are no other relevant points between $z_{i-1}$ and $z_{i}$. (This can be done by the same reasoning by which we excluded that $h^{*}\left(z_{i-1}=h^{*}\left(z_{i}\right)\right)$.) Then $z_{i-1} \in \omega_{i}^{-}$. As $h^{*} \equiv h^{*}\left(z_{i-1}\right)$ on $\beta_{i-1}^{+}$, we have $\beta_{i-1}^{+} \subset \omega_{i}^{-}$ and thus $w_{i-1}^{+}=\beta_{i-1}^{+} \cap \gamma \in \omega_{i}^{-} \cap \partial E_{i-1}$. Hence, by choosing $W_{i}^{-}=w_{i-1}^{+}$in part (a) of the proof, we obtain $\zeta_{i-1} \subset E_{i-1} \cap \gamma \preceq w_{i-1}^{+}=W_{i}^{-} \prec \eta_{i} \prec w_{i}^{-}$, i.e. we have accomplished all the requirements.

Remark 16. We emphasize that - as a by-product of the preceding proof - we have that, if $z_{i-1}$ is an extremum point, then $E_{i-1} \cap \zeta_{i}=\varnothing$, whatever $z_{i}$ is. This will be the key to proving Theorem 3 .

In order to prove Theorem 3 we need some other preliminaries. We carry out this part of our analysis on the strip S. Let $z_{i}$ be a maximum point for $h^{*}$, with $h^{*}\left(z_{i}\right)=a>0$. We can construct $E_{i}$ as in the preceding proof (see Fig. 2). We consider the component $F$ of the set $\left\{k^{*}>0\right\}$ containing $\beta_{i}^{-}$and its boundary $\partial F$ relative to $\bar{\Omega}$. The component of $\partial F$ containing $z_{i}$ is an analytic curve $\alpha$ that meets $\gamma$ (it can be proved in the same way as for the component $\left.\omega^{-}\left(z_{0}, a\right)\right)$. In order to evaluate $h$, we have to evaluate $|\nabla u|$, or $\rho$ (recall that $\left.\rho=-\log |\nabla u|\right)$. It holds that $\rho\left(z_{i}\right)=0$ and $\mathrm{d} \rho=(\partial \theta / \partial u) \mathrm{d} v-\frac{\partial \theta}{\partial v} \mathrm{~d} u$. The latter can be rewritten as $\mathrm{d} \rho=-h^{*} \mathrm{~d} u$ on the curve $\alpha$ (see Proposition 9). By the Cauchy-Riemann equations, $h^{*}$ is strictly increasing on $\alpha$ oriented from $L$ to $l$; therefore we can apply the following lemma, whose proof can be found in Appendix B.

Lemma 17. Let $h^{*}$ be strictly increasing on an arc $\alpha$ joining $L$ to $l$. If $h^{*}=a$ at the point in which $\alpha$ and $L$ meet, then

$$
\int_{\alpha} h^{*} \mathrm{~d} u \geq a .
$$

Thus we obtain

Proposition 18. Let $z_{i} \in \Gamma$ be a maximum point for $h^{*}$ restricted to $\Gamma$, with $h^{*}\left(z_{i}\right)=a \geq 0$. Then

$$
h\left(z_{i}^{1}\right)>a \mathrm{e}^{a},
$$

where $z_{i}^{1}$ is the point at which $\alpha$ and $l$ meet.

Proof. Put $z_{i}^{1}=\alpha \cap l$; then we have

$$
h\left(z_{i}^{1}\right)=h^{*}\left(z_{i}^{1}\right) \cdot \mathrm{e}^{-\rho\left(z_{i}^{1}\right)}>h^{*}\left(z_{i}\right) \cdot \mathrm{e}^{-\rho\left(z_{i}^{1}\right)} \geq h\left(z_{i}\right) \cdot \mathrm{e}^{h\left(z_{i}\right)} .
$$

The first inequality is a consequence of $h^{*}$ being increasing on $\alpha$; the second one comes from Lemma 17 . 
We can now prove Theorem 3.

Proof (Proof of Theorem 3). Our hypothesis regarding $h$ restricted to $\Gamma$ is readily translated into a hypothesis concerning $h^{*}$, since $h \equiv h^{*}$ on $\Gamma$.

We go through the proof of Theorem 1, up to obtaining the sets $E_{i} \cap \gamma$ for the non-negative maximum points, and the $\operatorname{arcs} \zeta_{i}$ for all the other $z_{i}$.

For each non-negative maximizer $z_{i}$, we proceed to determine $z_{i}^{1}$ as above, exploiting the level lines $\{k=0\}$. We have $z_{i}^{1} \in E_{i} \cap \gamma$. By the previous remark we know that $z_{i}^{1} \notin \zeta_{i+1}^{1}$ and by Proposition 18, inequality (7) holds. Choosing as $\zeta_{i}^{1}$ the maximal subset containing $z_{i}^{1}$ and such that $h^{*}$ is constant we complete the proof.

If we apply the same technique to the case of non-positive minimizers, we obtain that

$$
h\left(z_{i}^{1}\right)=h^{*}\left(z_{i}^{1}\right) \cdot \mathrm{e}^{-\rho\left(z_{i}^{1}\right)}\left\{\begin{array}{l}
<h\left(z_{i}\right) \cdot \mathrm{e}^{-\rho\left(z_{i}^{1}\right)}, \\
\geq h^{*}\left(z_{i}^{1}\right) \cdot \mathrm{e}^{h\left(z_{i}\right)} .
\end{array}\right.
$$

Remark 19. Suppose $z_{i}$ is a positive maximizer for $h$ restricted to $\Gamma$. As we have seen, $h^{*}$ is strictly increasing on $\alpha$, so $\alpha \subset \omega^{+}\left(z_{i}, 0\right)$. If $\Gamma_{i}=\omega^{+}\left(z_{i}, 0\right) \cap \Gamma$ and we choose $\gamma_{i}$ to be the component of $\omega^{+}\left(z_{i}, 0\right) \cap \gamma$ to which $z_{i}^{1}$ belongs, we have $\max \left\{h(z): z \in \gamma_{i}\right\} \geq h\left(z_{i}^{1}\right)>\max \left\{h(z): z \in \Gamma_{i}\right\}=h\left(z_{i}\right)$. Therefore the arc $\gamma_{i}$ bends more than $\Gamma_{i}$ does.

\section{Appendix A. Proof of Lemma 12}

We recall that both $\Gamma$ and $\gamma$ are Jordan curves, i.e. they are homeomorphic to the unit circle. Therefore the statements concerning the decomposition of $\Gamma$ follow.

Suppose first that

$$
\Gamma \cap c=\left\{z_{1}, z_{2}\right\} .
$$

Then $c \backslash\left\{z_{1}, z_{2}\right\} \subset D_{b}(\Gamma) \backslash D_{b}(\gamma)$ and $\left\{z_{1}, z_{2}\right\} \subset \Gamma$. Hence $c$ is a crosscut in $D_{b}(\Gamma)$ (see Newman's treatise [11, Ch. 5 Par. 2, Th. 11.7]) and so $D_{b}(\Gamma)$ is divided by it into two components $A_{1}, A_{2}$ such that $A_{1} \cap A_{2}=\varnothing$, $A_{1} \cup A_{2}=D_{b}(\Gamma) \backslash c$. As $D_{b}(\gamma) \subset D_{b}(\Gamma)$ and $c \subset \bar{\Omega}$ we have $D_{b}(\gamma)=\left(D_{b}(\gamma) \cap A_{1}\right) \cup\left(D_{b}(\gamma) \cap A_{2}\right)$ and just one of $D_{b}(\gamma) \cap A_{i}$ is not empty.

If one or more sub-arcs of $c$ lie on $\Gamma$ we can still apply the above reasoning iteratively to each arc that satisfies (A.1), up to identifying the component containing $D_{b}(\gamma)$.

\section{Appendix B. Proof of Lemma 17}

Let $\mathrm{S}=\{v+\mathrm{i} u: 0<u<1\}, L=\{v+\mathrm{i} u: u=0\}, l=\{v+\mathrm{i} u: u=1\}$ and $c(t)=v(t)+\mathrm{i} u(t):[a, b] \rightarrow \overline{\mathrm{S}}$ be such that there exists $m \in(a, b)$ such that for all $t \in[a, m] u^{\prime}(t) \leq 0$, for all $t \in[m, b] u^{\prime}(t) \geq 0$. Put $\Delta u=u(b)-u(a)$.

Suppose $f$ is a piecewise $C^{1}$ function which is increasing on $c$ and $f(c(a))=A$. Then by direct computation we obtain

$$
\int_{c} f \mathrm{~d} u \geq f(c(m)) \cdot \Delta u
$$

If $\Delta u \geq 0(\Delta u \leq 0)$ it follows that $\int_{c} f \mathrm{~d} u \geq f(c(a))\left(\int_{c} f \mathrm{~d} u \geq f(c(b))\right)$.

Now assume that $d$ is a curve obtained by joining up curves like $c$ above, all the increments having the same sign: Let $d(t)=v(t)+\mathrm{i} u(t):[a, b] \rightarrow \overline{\mathrm{S}}$ be a continuous curve, $f$ be increasing on $d$. Suppose there exist $d_{i}(t): v_{i}(t)+\mathrm{i} u_{i}(t):\left[a_{i}, b_{i}\right] \rightarrow \overline{\mathrm{S}}, i=1, \ldots n$, such that $d=\bigcup_{i=1}^{n} d_{i},[a, b]=\bigcup_{i=1}^{n}\left[a_{i}, b_{i}\right], a_{i}=b_{i-1}$ for $i=2, \ldots n$, and suppose that for each $i \in\{1, \ldots, n\}$ there exists a $m_{i} \in\left[a_{i}, b_{i}\right]$ such that for all $t \in\left[a_{i}, m_{i}\right], u_{i}^{\prime} \leq 0$, for all $t \in\left[m_{i}, b_{i}\right], u_{i}^{\prime} \geq 0$.

Put for each $i \in\{1, \ldots, n\}, \Delta^{i} u=d_{i}\left(b_{i}\right)-d_{i}\left(a_{i}\right)$. Then by a straight inductive argument, we obtain that if for all $i \in\{1, \ldots, n\}$ it holds that $\Delta^{i} u \geq 0$, or $\Delta^{i} u \leq 0$ then

$$
\int_{d} f \mathrm{~d} u \geq f(d(a))
$$


or

$$
\int_{d} f \mathrm{~d} u \geq f(d(b))
$$

respectively.

Now let us take up the relevant case. We decompose the curve $\alpha(t)=v(t)+\mathrm{i} u(t)$ as a sum of curves like $d$ above. Let $\mathcal{C}=\left\{\mathcal{I}_{i}\right\}_{i=1 \ldots 2 n+1}$ (for a suitable $n \in \mathbb{N}$ ) be a collection of intervals such that $[0,1]=\bigcup_{i=1}^{2 n+1} \mathcal{I}_{i}$, and $\dot{\mathcal{I}}_{i_{1}} \cap \stackrel{\circ}{\mathcal{I}}_{i_{2}}=\varnothing$ whenever $i_{1} \neq i_{2}$. Moreover we require each $\mathcal{I}_{i}$ to be maximal with respect to the statement: either $u^{\prime} \geq 0$ in $\mathcal{I}_{i}$ or $u^{\prime} \leq 0$ in $\mathcal{I}_{i}$. Put for each $i \in\{1, \ldots 2 n+1\}, \mathcal{I}_{i}=\left[a_{i}, b_{i}\right]$. We remark that on account of the geometry of $\overline{\mathrm{S}}$, on $\mathcal{I}_{2 n+1}$ and $\mathcal{I}_{1}$ we have $u^{\prime} \geq 0$ (recall that $\alpha\left(b_{2 n+1}\right) \in l, \alpha\left(a_{1}\right) \in L$ ) and so $u^{\prime} \geq 0$ on $\mathcal{I}_{i}$ for all odd $i$, while for even $i$ we have $u^{\prime} \leq 0$ (this also amounts to a justification for $\mathcal{C}$ having an odd number of elements).

Now for each even $k \in\{2, \ldots, 2 n\}$, put $\mathcal{J}_{\frac{k}{2}}=\mathcal{I}_{k} \cup \mathcal{I}_{k+1}$ and then $\mathcal{J}_{0}=\mathcal{I}_{1}$. Then each $\alpha_{\mid \mathcal{J}_{j}}$ is a curve of the same kind as $c$ above. For each $j \in\{1, \ldots, n\}$ put $\Delta^{i} u=\int_{\mathcal{J}_{j}} u^{\prime}$. On the set $\mathcal{D}=\left\{\mathcal{J}_{j}\right\}_{j=1 \ldots n}$ (we leave out $\mathcal{J}_{0}$ for a while) define an equivalence relation (symbol: $\simeq$ ) as

$$
\mathcal{J}_{j_{1}} \simeq \mathcal{J}_{j_{2}} \Longleftrightarrow \begin{aligned}
& \text { for each } j: \min \left\{j_{1}, j_{2}\right\} \leq j \leq \max \left\{j_{1}, j_{2}\right\} \\
& \operatorname{sgn}\left(\Delta^{j_{1}} u\right)=\operatorname{sgn}\left(\Delta^{j} u\right)=\operatorname{sgn}\left(\Delta^{j_{2}} u\right)
\end{aligned}
$$

Therefore we have taken a partition of $\mathcal{D}$ into sub-collections $\left\{\mathcal{B}_{\varepsilon}\right\}$. Each $\mathcal{B}_{\varepsilon}$ is formed by adjoining intervals $\mathcal{J}_{j}$ all sharing the sign of the increment $\Delta^{j} u$. Thus each $\alpha_{\mid \cup \mathcal{B}_{\varepsilon}}$ is a curve like $d$ above.

We go through induction on the number of the collections $\mathcal{B}_{\varepsilon}$ to prove that for all $v \in \mathbb{N}$,

$$
\int_{\bigcup_{\varepsilon=1}^{v} \alpha\left(\cup \mathcal{B}_{\varepsilon}\right)} f \mathrm{~d} u \geq f\left(\alpha\left(A_{\nu}\right)\right) \cdot \sum_{\varepsilon=1}^{v} \delta^{\varepsilon} u
$$

where for each $\varepsilon \in\{1, \ldots, v\}, \delta^{\varepsilon} u=\int_{\bigcup \mathcal{B}_{\varepsilon}} u^{\prime}=\sum_{j: \mathcal{J}_{j} \in \mathcal{B}_{\varepsilon}} \Delta^{j} u$, and $\left[A_{v}, B_{v}\right]=\bigcup \mathcal{B}_{v}=\bigcup\left\{\mathcal{J}_{j}: \mathcal{J}_{j} \in \mathcal{B}_{v}\right\}$.

In order to take advantage of the fact that $u^{\prime} \geq 0$ close to $\alpha(1)$, we suppose we have numbered the collections $\mathcal{B}_{\varepsilon}$ so that $\alpha(1) \in \mathcal{B}_{1}$ and $\varepsilon$ growing while moving backwards along $\alpha$, towards $\alpha(0)$. Then we can observe that the inductive case $v=1$ is a straight application of what we have shown to hold for the curve $d$ above, since $\delta^{1} u \geq 0$.

Now suppose (B.2) holds for $v=N-1$, and try to prove (B.2) for $v=N$, i.e. the case $\left\{\mathcal{J}_{j}: j=1 \ldots n\right\}=$ $\bigcup_{\varepsilon=1}^{N} \mathcal{B}_{\varepsilon}$. We have

$$
\int_{\bigcup_{\varepsilon=1}^{N} \alpha\left(\cup \mathcal{B}_{\varepsilon}\right)} f \mathrm{~d} u=\int_{\bigcup_{\varepsilon=1}^{N-1} \alpha\left(\cup \mathcal{B}_{\varepsilon}\right)} f \mathrm{~d} u+\int_{\alpha\left(\cup \mathcal{B}_{N}\right)} f \mathrm{~d} u \geq f\left(\alpha\left(A_{N-1}\right)\right) \cdot \sum_{\varepsilon=1}^{N-1} \delta^{\varepsilon} u+\int_{\alpha\left(\cup \mathcal{B}_{N}\right)} f \mathrm{~d} u,
$$

where the inequality is a consequence of induction. It may happen that $\delta^{N} u \geq 0$ or $\delta^{N} u \leq 0$. Before dealing with these alternatives, we observe that we have

$$
\sum_{\varepsilon=1}^{N} \delta^{\varepsilon} u \geq 0
$$

(otherwise we would have $\alpha(1)>1$ ).

When $\delta^{N} u \geq 0$, we can extend (B.3) by

$$
\geq f\left(\alpha\left(A_{N-1}\right)\right) \cdot \sum_{\varepsilon=1}^{N-1} \delta^{\varepsilon} u+f\left(\alpha\left(A_{N}\right)\right) \cdot \delta^{N} u \geq f\left(\alpha\left(A_{N}\right)\right) \cdot \sum_{\varepsilon=1}^{N} \delta^{\varepsilon} u,
$$

the first inequality following from (B.1a), the second from $f$ being monotonic and (B.4).

When $\delta^{N} u \leq 0$, we extend (B.3) as

$$
\geq f\left(\alpha\left(A_{N-1}\right)\right) \cdot \sum_{\varepsilon=1}^{N-1} \delta^{\varepsilon} u+f\left(\alpha\left(A_{N-1}\right)\right) \cdot \delta^{N} u=f\left(\alpha\left(A_{N-1}\right)\right) \cdot \sum_{\varepsilon=1}^{N} \delta^{\varepsilon} u \geq f\left(\alpha\left(A_{N}\right)\right) \cdot \sum_{\varepsilon=1}^{N} \delta^{\varepsilon} u
$$


The first inequality is a consequence of (B.1b); the second from $f$ is monotonic from (B.4).

We still have to take into account $\mathcal{J}_{0}$, but that does not do any harm. Indeed

$$
\begin{aligned}
\int_{\alpha} f \mathrm{~d} u & =\int_{\bigcup_{\varepsilon=1}^{N} \alpha\left(\bigcup \mathcal{B}_{\varepsilon}\right)} f \mathrm{~d} u+\int_{\alpha\left(\mathcal{J}_{0}\right)} f \mathrm{~d} u \geq f\left(\alpha\left(A_{N}\right)\right) \cdot \sum_{\varepsilon=1}^{v} \delta^{\varepsilon} u+f(\alpha(0)) \cdot \Delta^{0} u \\
& =f\left(\alpha\left(A_{\nu}\right)\right) \cdot \sum_{j=1}^{n} \delta^{j} u+f(\alpha(0)) \cdot \Delta^{0} u \geq f(\alpha(0)) \cdot \sum_{j=0}^{n} \Delta^{j} u=f(\alpha(0)) .
\end{aligned}
$$

\section{References}

[1] M. Flucher, M. Rumpf, Bernoulli's free-boundary problem, qualitative theory and numerical approximation, J. Reine Angew. Math. 486 (1997) $165-204$.

[2] A. Beurling, Free boundary problems for the Laplace equation, vol. 1, Institute for Advanced Study Seminar, 1957, pp. 248-263. Republished in Arne Beurling Collected Works etc.

[3] A. Acker, On the geometric form of free boundaries satisfying a Bernoulli condition, Math. Methods Appl. Sci. 6 (4) (1984) $449-456$.

[4] A. Acker, On the geometric form of free boundaries satisfying a Bernoulli condition. II, Math. Methods Appl. Sci. 8 (3) (1986) $387-404$.

[5] H. Lewy, A note on harmonic functions and a hydrodynamical application, Proc. Amer. Math. Soc. 3 (1952) 111-113.

[6] H.W. Alt, L.A. Caffarelli, Existence and regularity for a minimum problem with free boundary, J. Reine Angew. Math. 325 (1981) $105-144$.

[7] G. Talenti, On functions, whose lines of steepest descent bend proportionally to level lines, Ann. Sc. Norm. Super. Pisa Cl. Sci. (4) 10 (4) (1983) 587-605.

[8] D.E. Tepper, Free boundary problem, SIAM J. Math. Anal. 5 (1974) 841-846.

[9] G. Alessandrini, R. Magnanini, The index of isolated critical points and solutions of elliptic equations in the plane, Ann. Sc. Norm. Super. Pisa Cl. Sci. (4) 19 (4) (1992) 567-589.

[10] B.A. Dubrovin, A.T. Fomenko, S.P. Novikov, The Geometry of Surfaces, Transformation Groups, and Fields, Springer, 1992.

[11] M.H.A. Newman, Elements of the Topology of Plane Sets of Points, Cambridge University Press, Cambridge, 1951. 